This item was submitted to Loughborough's Research Repository by the author.

Items in Figshare are protected by copyright, with all rights reserved, unless otherwise indicated.

\title{
High throughput volatile fatty acid skin metabolite profiling by thermal desorption secondary electrospray ionisation mass spectrometry
}

\section{PLEASE CITE THE PUBLISHED VERSION}

http://dx.doi.org/10.1039/c4an00134f

\section{PUBLISHER}

(C) The Royal Society of Chemistry

\section{VERSION}

AM (Accepted Manuscript)

\section{PUBLISHER STATEMENT}

This work is made available according to the conditions of the Creative Commons Attribution-NonCommercialNoDerivatives 4.0 International (CC BY-NC-ND 4.0) licence. Full details of this licence are available at: https://creativecommons.org/licenses/by-nc-nd/4.0/

\section{LICENCE}

CC BY-NC-ND 4.0

\section{REPOSITORY RECORD}

Martin, Helen J., James C. Reynolds, Svetlana Riazanskaia, and C.L. Paul Thomas. 2019. "High Throughput Volatile Fatty Acid Skin Metabolite Profiling by Thermal Desorption Secondary Electrospray Ionisation Mass Spectrometry". figshare. https://hdl.handle.net/2134/16053. 


\section{HIGH THROUGHPUT VOLATILE FATTY ACID SKIN METABOLITE PROFILING BY THERMAL DESORPTION SECONDARY ELECTROSPRAY IONISATION MASS SPECTROMETRY.}

Helen J Martin¹, James C Reynolds ${ }^{1}$, Svetlana Riazanskaia,2, 'C. L. Paul Thomas ${ }^{1}$.

1. Centre for Analytical Science, Department of Chemistry, Loughborough University, Loughborough, Leicestershire. LE11 3TU. UK

2 Unilever R\&D, Port Sunlight, Quarry Road East, Bebington, Wirral, UK.

\section{ABSTRACT}

The non-invasive nature of volatile organic compound (VOC) sampling from skin makes this a priority in the development of new screening and diagnostic assays. Evaluation of recent literature highlights the tension between the analytical utility of ambient ionisation approaches for skin profiling and the practicality of undertaking larger campaigns (higher statistical power), or undertaking research in remote locations. This study describes how VOC may be sampled from skin and recovered from a polydimethylsilicone sampling coupon and analysed by thermal desorption (TD) interfaced to secondary electrospray ionisation (SESI) time-of-flight mass spectrometry (MS) for the high throughput screening of volatile fatty acids (VFAs) from human skin.

Analysis times were reduced by $79 \%$ compared to gas chromatography-mass spectrometry methods (GC-MS) and limits of detection in the range 300 to $900 \mathrm{pg} \mathrm{cm}^{-2}$ for VFA skin concentrations were obtained.

Using body odour as a surrogate model for clinical testing 10 Filipino participants, 5 high and 5 low odour, were sampled in Manilla and the samples returned to the UK and screened by TD-SESI-MS and TD-GC-MS for malodour precursors with greater than $>95 \%$ agreement between the two analytical techniques. Eight additional VFAs were also identified by both techniques with chains 4 to 15 carbons long being observed. TD-SESIMS appears to have significant potential for the high throughput targeted screening of volatile biomarkers in human skin.

•c.l.p.thomas@Iboro.ac.uk 


\section{INTRODUCTION}

Analysis of the volatile organic compound (VOC) profile of human skin is an alternative, non-invasive, approach to invasive blood-based methodologies for diagnosis and studying human function. VOCs observed on and in skin are derived from: glandular secretions; perfusion from underlying blood vessels; products of micro-biological metabolism; and exogenous components (Environmental contamination and personal care products for example) [1-2]. The highly individualised and dynamic nature of these profiles, and the utility of skin profiling have been reviewed [3].

Sampling techniques for skin VOC profiles include: whole sweat collection [4-5]; liquid extraction [6-7]; adsorbent materials placed in direct contact with the skin surface [2, 8,14] or headspace approaches [6, 9, 15-16]. Analysis of skin has identified many types of VOC, and the list includes: volatile fatty acids (VFAs), aldehydes, alcohols, aliphatic and aromatic hydrocarbons, amides, amines, esters, halides, ketones and volatile sulphur compounds (VSCs) [2-3]. Multi-variate analysis (MVA) indicates gender specific information within such profiles $[13,17]$. Studies of skin VOC profiles have identified mosquito attractants [11-12] and monitored fragrance release [9-10]. Compounds associated with chronic wounds [18] as well as signatures differentiating between melanoma, naevi and healthy skin [19] have been reported. Skin profiling has also been applied to ammonia monitoring; non-invasive headspace capture of ammonia from skin enabled such an approach to be compared against blood gas levels. These measures were correlated $(R=0.84, p<0.01)$ and the approach was extended to proof-of-concept screening for hepatic disease with median levels of ammonia release from the skin of subjects with liver disease estimated to be ca. $3 \mathrm{ng} \mathrm{cm}-2(\mathrm{~N}=24)$, compared to ca. $2 \mathrm{ng}$ $\mathrm{cm}^{-2}(\mathrm{~N}=24)$ in healthy controls [20].

The foundations for such skin profiling studies are to be found in research on body odour. Individual skin profiles have been developed as 'barcodes' of scent for forensic [21] and diagnostic application [22], and gender and age specific signatures have been proposed as contributing factors to an individual's profile [23-26]. Olfactory analysis, either in-vivo or by organoleptic analysis of chromatographic eluents [27], combined with analytical measurement have established levels of odour to correlate with VSCs and VFAs, with 3-methyl-2-hexenoic acid cited as a critical molecular factor [26-28]. Finally, the quantity of anecdotal evidence of canine olfaction of disease, increasingly supported 
by scientific studies [29-32], reinforces the proposition of non-invasive skin VOC profiling for diagnosis and condition monitoring.

Direct contact and headspace skin sampling devices generally employ thermal desorption (TD) for the recovery of VOCs which is interfaced to gas chromatography (GC), mass spectrometry (MS) [3]. Such approaches generate extensive VOC profiles that are rich in information, and are potentially useful for global profiling and non-targeted biomarker discovery. However, such analytical workflows are time consuming, and an analysis time of more than an hour per sample mitigates against the large sample numbers needed to validate markers and is too long for high throughput analysis or clinical screening applications.

"Ambient" mass spectrometry approaches, where samples are introduced directly to an external ionisation source, for skin analysis provide rapid and sensitive analysis. Mass spectral "fingerprints" obtained in-vivo from skin with desorption electrospray ionisation (DESI) [33], secondary electrospray ionisation (SESI) [34-35] and extractive electrospray ionisation (EESI) [36] are exciting and promising developments. Selected ion flow tube mass spectrometry (SIFT-MS) has provided real-time information for acetone emanating from skin [37] and proton transfer reaction mass spectrometry (PTR-MS) has been applied to monitoring of lipid peroxidation products and the fatty acid composition of skin [38]. All these methods enable fast, selective and sensitive analysis of the area of a participant's skin presented to the instrument. These approaches, whilst offering a distinct time advantage, require the participant to present a part of their body to the instrument, which is not always practical. Further, consider for example, the practicalities of sampling and analysing a lot of participants' skin over a short period. The difficulty increases if the study encompasses a large geographic region, or remote locations. Clinical safety considerations and risk assessments also require instruments and systems to be sterile before use with a patient; repeatedly dousing sensitive instruments for VOC analysis with disinfectant is a problematic protocol.

Recently thermal desorption secondary electrospray ionisation-ion mobility-mass spectrometry (TD-SESI-IM-MS) was proposed for rapid breath screening as an alternative to TD-GC-MS. The potential for rapidly generating high-fidelity mass spectra of exhaled breath VOC without a lengthy chromatography step was demonstrated [39], and the current work is informed by this approach, with the aim of describing the targeted analysis of VFAs present in and on human skin. 
Sampling skin with polydimethylsiloxane (PDMS) coupons $\left(0.5 \mathrm{~cm}^{2} 0.5 \mathrm{~mm}\right.$ thick) placed on the skin surface with analysis by thermal desorption gas chromatography mass spectrometry has been proposed for targeted and non-targetted analysis of VOC skin metabolites and catabolites. The relative standard deviation (RSD) of this approach varies with analyte volatility, with values between 7 and 19\% observed during in-vitro tests. In-vivo, RSD values up to a maximum of $32 \%$ have been observed; for trace levels of a methylated hydrocarbon tentatively assigned to 3,7-dimethyloct-1-ene, and limits of detection were estimated to be of the order of 50pg [1]. The sensitivity, reproducibility, comfort and ease-of-use of a PDMS skin-patch sampler makes for a practical and straightforward methodology for undertaking more extensive studies on human skin, either in clinic or in the field.

The current research combined the sampling attributes of a 'skin patch' with thermal desorption secondary electrospray ionisation-mass spectrometry (TD-SESI-MS) [39]. TDSESI-MS was found to have limits of detection no higher than $1 \mathrm{ng}$ per sample, with an in-vivo RSD of $13.5 \%$. The most relevant analytical characteristic to this study was a mass accuracy of $1.4 \mathrm{ppm}$ enabling metabolite identities to be assigned with a high level of confidence. The combination of skin patch samples with TD-SESI-MS provides a highthroughput, clinically compatible and scalable methodology for targeted metabolite/VOC analysis of human skin.

The aim of this study was to evaluate the potential of skin patch sampling with TD-SESIMS analysis for phenotypic screening, potential diagnostic applications and characterisation of skin metabolism. To do this a panel of volunteer participants was recruited, with half of them having a genetic trait relating to "body odour". The specific hypothesis was that a single nucleotide polymorphism (SNP), $538 \mathrm{G} \rightarrow \mathrm{A}$, caused a G180R substitution in the ABCC11 gene that would result in reduced concentrations of apocrine derived axillary odour precursors, with special emphasis on 3-methyl-2-hexenoic acid [4041]. In other words half of our participants would have the genetic attribute of apocrine secretions that did not contain 3-methyl-2-hexenoic acid; they did not have a characteristic "sweaty smell". Five other skin metabolite VFA's were also included in the study (butanoic acid, 2 methylpropanoic acid, pentanoic acid, 3-methylbutanoic acid and hexanoic acid). These analytes were not expected to be affected as strongly by the SNP and their presence in the screen would indicate that the absence of 3-methyl-2-hexenoic acid was not attributable to a measurement artefact and a reduction in the efficiency of 
analyte recovery. The resultant data were to be compared to those derived from a thermal desorption gas chromatograph electron ionisation time-of-flight mass spectrometer (TD-GC-ToFMS) and an organoleptic assessment of malodour.

\section{EXPERIMENTAL}

\section{Ethics and Participant Preparation}

This research was conducted in accordance with the ethical principles of Good Clinical Practice and the Declaration of Helsinki. The local ethics committee approved the study before commencement of the work, and all subjects gave written informed consent. Caucasians and Africans possess a strong axillary distinctive smell, whereas many Asians don't. There is evidence that the gene $A B C C 11$ is an important factor in the formation of axillary odour. Further recent studies have proposed that a single-nucleotide polymorphism (SNP) 538G $\rightarrow$ A, leads to individuals having no characteristic axillary odour. This SNP is prominent among Asian people, hence a consumer test centre in Manila was selected as the sampling point. [40, 41]. Healthy female subjects, aged between 20 and 55 years of age were recruited for this study, following a qualifying medical questionnaire. Participants on medication, suffering from any skin disorder or systemic disease or if with a history of being sensitive to underarm personal care products were excluded.

Participants were requested not to use antiperspirant or deodorant products in the 3 week pre-test period, and instructed to wash their underarm area with nothing but unfragranced liquid soap. All samples were taken at the Unilever Consumer Studies Centre (Manila, Philippines).

\section{Sampling}

The PDMS skin sampling patches (Goodfellow Cambridge Limited) were prepared for use by cleaning with ultrasonic washing in methanol at $30^{\circ} \mathrm{C}$ and then individually placed inside clean, empty thermal desorption tubes. These were then heated to $185^{\circ} \mathrm{C}$ under vacuum for 15 hours. Final conditioning of the patches involved purging with purified helium at $50 \mathrm{~cm}^{3} \mathrm{~min}^{-1}$ and $185{ }^{\circ} \mathrm{C}$ for 20 minutes. Each prepared skin patch was analysed by (TD-GC-MS) to verify that it was free from contamination. The thermal desorption tube containing the skin sampling patch was then removed from the thermal desorber and immediately sealed with Swagelok caps and shipped from Loughborough University to Manila in the Philippines where the samples were taken. 
Prior to VOC sampling, the intensity of the participants' axillary odour was evaluated organoleptically by six independent assessors against a set of standard solutions of the target VFAs and assigned a malodour score from 1 to $5 ; 1$ being the weakest discernable odour and 5 the most intense. The mean of the four scores was taken as the mean malodour score (MMS) for the participant. In this pilot study 5 participants with high a organoleptic assessment of VFA odour (MMS 3 to 5 ) and 5 participants with low organoleptic assessments (MMS 1 - 2.5) were selected.

A VFA skin sample was obtained from each participants' axilla with a prepared skin patch that was removed from a sealed thermal desorption tube and immediately placed on the skin surface and covered with a cotton pad (conditioned previously under vacuum at $70^{\circ} \mathrm{C}$ for 15 hours and then stored in an air tight package until use). The skin sampling patch was left in place for 30 min before it was removed and immediately resealed inside the thermal desorption tube [1], which was then rapidly cooled to $-80{ }^{\circ} \mathrm{C}$ and returned to the laboratory for analysis.

Instrumentation

Thermal Desorption Secondary Electrospray Ionisation Time-of-Flight Mass Spectrometry (TD-SESI-MS )

The modification of an electrospray source to TD-SESI-MS has been described elsewhere [40]. Briefly, the outlet transfer line $(0.25 \mathrm{~mm}$ i.d deactivated fused silica) from a thermal desorption unit (Markes International UNITY 1) was interfaced to the electrospray source of an ion mobility-quadrupole time-of-flight mass spectrometer (Waters Synapt) operating with the ion mobility off.The front of the ionisation source was removed along with the lockspray baffle plate and the reference sprayer assembly to accommodate the heated shroud of the transfer line and allow accurate positioning of the end of the capillary. The capillary end was aligned 0.5 to $1 \mathrm{~cm}$ from the sample cone of the mass spectrometer and $5 \mathrm{~mm}$ from the electrospray emitter. This ensured that the desolvation gas focused the gaseous sample stream from the capillary tip towards the sample cone.

It is important to note that this procedure required electrical isolation switches within the ionisation assembly to be defeated. Physical barriers, warning signs and exclusion areas were used to reduce the risk from this electrical hazard.

Analysis of the skin sampling patches entailed $10 \mathrm{~min}$ thermal desorption at $180^{\circ} \mathrm{C}$ with a Helium flow of $6 \mathrm{~cm}^{3} \mathrm{~min}^{-1}$. The desorbed products were concentrated in a cold trap held at $-10^{\circ} \mathrm{C}$ and packed with a dual sorbent bed of Tenax and Carbograph-1TD 
(Markes International, U-T2GPH), injection to the SESI source involved ballistic heating of the "cold-trap" at $32^{\circ} \mathrm{C} \mathrm{s}^{-1}$ to $300{ }^{\circ} \mathrm{C}$. The "cold-trap" was maintained at $300^{\circ} \mathrm{C}$ for 5 min. The transfer line was maintained at $150^{\circ} \mathrm{C}$. An important aspect of the quality assurance of this process was the inclusion of blank runs before and after each sample to verify that the instrument was free of contamination and that no residual analyte remained in the sample coupon or within the analytical pathway.

The mass spectrometer was operated in negative mode with: a capillary voltage of $3 \mathrm{kV}$, a cone voltage of $10 \mathrm{~V}$, a source temperature of $120^{\circ} \mathrm{C}$, and a desolvation temperature of $150^{\circ} \mathrm{C}$. The desolvation gas was nitrogen supplied at a flow $5 \mathrm{dm}^{3} \mathrm{~min}^{-1}$. No cone gas was supplied in this study. The electrospray solvent was unbuffered 50/50 (v/v) methanol/water infused into the source at $5 \mu \mathrm{min}^{-1}$.

Thermal Desorption Gas Chromatography Time-of-Flight Mass Spectrometry (TD-GCToFMS)

Sample recovery was by thermal desorption (Markes International, UNITY 2) of the skin sampling patches interfaced to a GC-ToF-MS (Waters GCT Premier gas chromatograph time-of-flight mass spectrometer). Skin patches were thermally desorbed at $180^{\circ} \mathrm{C}$ for 10 min with a $50 \mathrm{~cm}^{3} \mathrm{~min}^{-1}$ helium flow to a cold trap held at $-10^{\circ} \mathrm{C}$. Injection from the cold trap was achieved by ballistic heating at $32^{\circ} \mathrm{C} \mathrm{s}^{-1}$ to $300{ }^{\circ} \mathrm{C}$. This temperature was maintained for $5 \mathrm{~min}$ with a $1 / 10$ split, the transfer line temperature was $150{ }^{\circ} \mathrm{C}$. The gas chromatograph was fitted with a $0.25 \mathrm{~mm}$ i.d., $60 \mathrm{~m}$ long capillary column with a stationary phase thickness of $2.5 \mu \mathrm{m}$. The stationary phase was a 5\% phenyl, 95\% methyl polysiloxane stationary phase (Cat no: 122-5562 Agilent DB-5MS), the temperature programme had an initial temperature of $40^{\circ} \mathrm{C}, 0 \mathrm{~min}$ hold, that increased at $5^{\circ} \mathrm{C} \mathrm{min}-1$ to $310^{\circ} \mathrm{C}$ where it was held for 6 minutes. The $\mathrm{GC}$ was operated at constant head pressure of $172 \mathrm{kPa}$ (25 psi). The electron impact source was operated in the positive mode at a temperature of $200^{\circ} \mathrm{C}$, with an electron energy of $70 \mathrm{eV}$. The mass spectrometer cycle time was $0.1 \mathrm{~s}$ (scan duration $0.09 \mathrm{~s}$, interscan delay $0.01 \mathrm{~s}$ ) with a mass-range of 40 to 550 .

Preparation of standards

Standard mixtures for evaluation of mean malodour score and instrument calibration of the target VFA metabolites were prepared. The stock solution contained butanoic acid ( $\left.4.8 \mu \mathrm{g} \mathrm{cm}^{-3}\right)$, 2-methylpropanoic acid acid $\left(4.8 \mu \mathrm{g} \mathrm{cm}^{-3}\right)$, pentanoic acid $\left(17 \mu \mathrm{gm}^{-3}\right), 3-$ methylbutanoic acid $\left(17 \mu \mathrm{gm}^{-3}\right)$, hexanoic acid $\left(10 \mu \mathrm{g} \mathrm{cm}^{-3}\right)$ and 3-methyl-2-hexenoic 
acid $\left(100 \mu g \mathrm{~cm}^{-3}\right)$ all dissolved in high purity methanol. This stock solution was designated to have an organoleptic mean malodour score (MMS) of 5; a strong sensation of malodour for most humans. Lesser strength solutions to mimic MMS 4 and below were then prepared by sequential 4-fold dilutions.

$5 \mu \mathrm{l}$ aliquots, the approximate volume of a droplet of an apocrine secretion [1], of the MMS 3 mixture were directly deposited onto blank skin patches in thermal desorption tubes during the thermal desorption and electrospray optimisation studies. Each of these aliquots contained $1.5 \mathrm{ng}$ of butanoic and 2-methylpropanoic acid, $5 \mathrm{ng}$ of pentanoic and 3-methylbutanoic acid, 3 ng of hexanoic acid and 30 ng 3-methyl-2-hexenoic acid.

\section{RESULTS AND DISCUSSION}

Approximately 600 mass spectrometric peaks were isolated from each sample in the negative mode over a 4 min profile, see the bottom trace in Figure 1 obtained from a participant without the (SNP) $538 \mathrm{G} \rightarrow \mathrm{A}$ who had a high organoleptic mean malodour score (MMS) of 4. Individual compounds were resolved on the basis of selected ion mass spectrometry. Butanoic and 2-methylpropanoic acid yield isobaric ions, as do pentanoic and 3-methylbutanoic acid and these pairs of isomers were combined. This gave four VFA targets that were designated as, C4 VFA (butanoic and 2-methylpropanoic), C5 VFA (pentanoic and 3-methylbutanoic), $\mathrm{H}$ (hexanoic) and 3M2H (3-methyl-2-hexenoic) acids.

Some separation between the VFA targets was observed in the thermal desorption profiles with C4 VFA (boiling point (BP) $164^{\circ} \mathrm{C} / 155^{\circ} \mathrm{C}$ ) eluting earliest followed by $\mathrm{C} 5$ VFA (BP $\left.186^{\circ} \mathrm{C} / 175^{\circ} \mathrm{C}\right)$, hexanoic acid (BP $203^{\circ} \mathrm{C}$ ) and 3-methyl-2-hexenoic acid (BP $225^{\circ} \mathrm{C}$ ) eluting last, Figure 1 . This partial separation introduced an element of selectivity into the sample introduction, and as such was thought to mitigate somewhat the effects of competitive ionisation from higher molecular weight skin matrix components.

Note that including the ion mobility function of the mass spectrometer was not helpful in this study for the resultant reduction in mass accuracy outweighed any selectivity benefits derived from a low-resolution T-wave ion mobility separation.

Limits of detection, determined from on-patch mass and signal-to-noise ratios were estimated to be no higher than: 500 pg for butanoic/2-methylpropanoic acid (C4 VFA); 900 pg for pentanoic/3-methylbutanoic acid (C5 VFA); 300 pg hexanoic acid (H); and 350 pg 3-methyl-2-hexenoic acid (3M2H). These levels were below the 1.9 ng odour threshold previously reported for 3-methyl-2-hexenoic acid [28]. 


\section{Skin Sample Analysis}

The thermal desorption tubes containing the skin sampling patches were removed from $80^{\circ} \mathrm{C}$ storage and warmed for $5 \mathrm{~min}$ at room temperature before loading into the thermal desorber for analysis. Figure 1 shows desorption profiles obtained from a skin sample provided by participant with an MMS $=4$, contrasted against a blank skin patch. The skin sample provided a complicated desorption profile with VOC components eluting for up to four minutes after the start of the ballistic heating of the cold trap. Figure 1 also shows the magnified (x 100) mass selected desorption profiles of the four target VFAs (C4 VFA, C5 VFA, H, and 3M2H). The partial separation, based on volatility, of the target VFAs is also evident.

\section{$<$ Figure 1 near here. $>>$}

Figure 2 contrasts the mass spectrum from the skin sample in Figure 1 against the mass spectrum of the blank skin patch and a number of background ions as well signals from the blank skin patch and the skin sample are evident. The most intense signal, $m / z$ 212.07, was present in all the samples and blanks and this was assigned to the plasticiser n-butyl benzensulfonamide, an ion that has been used previously as a lockmass in the negative ion mode [42] and was used in this study with the TD-SESI-MS data enabling the target VFAs Figure 2 to be identified with a mass accuracy of $3.5 \mathrm{ppm}$ or less [43].

The complexity of the VOC profile in, or on, skin was evident from the gas chromatography with many hundreds of resolved and unresolved components. The mass spectra from TD-SESI-MS also contain a significant degree of complexity, and it is helpful to note that competitive ionisation and matrix affects may result in the suppression of some signals. Nevertheless it was possible to corroborate the presence of the four VFA targets along with an extensive sequence of related VFA compounds with a total analysis times of 15 min compared to 70 minutes for TD-GC-MS. See Figure 3.

$<<$ Figure 2 \& 3 near here $>>$

Tables 1 and 2 compare the VFA targets isolated by the two methods alongside other non-targeted VFAs that were also identified. The efficacy of TD-SESI-MS for biomarker screening is apparent. The body malodour marker 3-methyl-2-hexenoic acid (3M2H) was observed in 4/5 high odour individuals and not at all in low odour individuals in both analytical techniques. The discriminating power of the TD-SESI-MS approach compared 
to TD-GC-MS was examined by performing a principle component analysis (PCA) on the data in Tables 1 and 2. An unsupervised 2 component PCA model of the TD-SESI-MS data (Table 1), $\mathrm{R}^{2}=0.993, \mathrm{Q}^{2}=0.868$ shown in Figure $4 \mathrm{a}$, reveals two clusters, one for high-odour and the other for low- odour individuals. The loading plot identified that the discrimination was based on the levels of 3-methyl-2-hexenoic $(3 \mathrm{M} 2 \mathrm{H})$ and hexanoic $(\mathrm{H})$ acids. Similarly, an unsupervised PCA model based on the TD-GC-MS data (Table 2), $\mathrm{R}^{2}=0.969, \mathrm{Q}^{2}=0.737$, see Figure $4 \mathrm{~b}$, also shows separation between the classes with the same VFA targets indicated through the loading plot.

$<<$ Figure 4 near here $>>$

PCA of the data from both techniques clustered Participant 4 with the low odour group, and this was not surprising for 3-methyl-2-hexenoic $(3 \mathrm{M} 2 \mathrm{H})$ was not detected by either technique. The organoleptic assessment scores for this participant were ambivalent. One assessor assigned a malodour score of 5 whilst the rest of the panel gave malodour scores between 2 and 3, with an overall MMS of 3.33. Excluding the score of 5 places participant 4 in a low odour category with a MMS of 2.5.

The data obtained from TD-SESI-MS and high fidelity TD-GC-MS was comparable and yielded equivalent phenotype classification based on the same molecular markers. Further examination of both data sets revealed higher molecular weight VFAs in, or on, skin. The high mass accuracy obtained by TD-SESI-MS enabled assignments to a series of VFA, Figure 2, and subsequent processing of the TD-GC-MS confirmed a series of VFAs from C8 to C15, Table 3 and Figure 3. These data are encouraging and indicate the potential utility of TD-SESI-MS for high-throughput targeted biomarker screening.

Figure 5 shows an example cumulative distribution function of the mass spectral peak intensities across the $\mathrm{m} / \mathrm{z}$ range 40 to 300 alongside the intensity distribution of the analytical features. A total of 598 mass spectral peaks were distributed across this range with $75 \%$ of the observed components present in the lowest $2.5 \%$ of the intensity range. The secondary electrospray mass spectra in the negative mode were information rich, and further metabolomic based investigations of such data in both positive and negative mode are a logical development of this work.

$<<$ Figure 5 near here $>>$ 
Qualitatively the TD-SESI-MS approach performed well alongside TD-GC-MS. VFA profiling by TD-SESI-MS was 79\% faster than TD-GC-MS and the potential for a high throughput targeted screening for biogenic VOCs appears promising. However, there are challenges to address to move this technique towards more quantitative protocols. The ubiquitous presence of siloxanes changes the ionisation chemistry and a build up of siloxanes within the analytical pathway was observed with increasing run numbers that disrupted the analysis if care was not taken to constantly monitor this phenomenon as part of the QA procedures. Development of this approach would usefully include further examination of the thermal desorption process and the transfer line as well as further study of the ionisation approach. The incorporation of ion mobility spectrometry with sufficient resolving power would enable isomer differentiation of the butanoic/2-methylpropanoic (C4 VFA) and pentanoic/3-methylbutanoic(C5 VFA) components and enable further study of structural isomers and enhance the spectral quality thorough the suppression of background interferences.

\section{CONCLUSIONS}

This research examined using skin patch sampling for high-throughput screening for phenotype markers. In this instance, four VFA targets associated with body malodour were sampled from the axilla of ten female participants, stored and transported from the Philippines to the UK, where they were analysed.

The four VFA target analytes were identified by accurate mass from skin samples with TD-SESI-MS with a maximum analysis time of $15 \mathrm{~min}$ and limits of detection estimated to be in the 100s $\mathrm{pg} \mathrm{cm}^{-2}$ region (A skin patch has a surface area of $0.5 \mathrm{~cm}^{2}$ ).

This study demonstrates the feasibility of adapting and adopting electrospray mass spectrometry systems for high-throughput profiling for VOC bio-markers from human skin samples. In this instance the focus of the research was to phenotypically classify individuals with a specific ((SNP) $538 \mathrm{G} \rightarrow \mathrm{A})$ in the human ABCC11 gene. As well as controlling body odour, ABCC11 is the subject of studies and much debate into breast cancer and drug resistance in cancer cells [44]. The underlying motivation in this research is the development of a suite of non-invasive measurements for personalised medicine. The current technique may be set-up with automated and un-attended batch analysis to process large numbers of non-invasive skin VOC samples, to generate clinical screening data from larger cohorts distributed across the world. The sampling methodology meets the most stringent requirements for bio-security and if necessary 
may be used on delicate skin structures to provide additional and complementary noninvasive metabolite and diagnostic data, perhaps on a par with breath profiling.

\section{ACKNOWLEDGEMENTS}

The authors gratefully acknowledge the support given to H.J.Martin by Unilever PLC and Waters Corporation in providing the GC-based analysis of the duplicate skin samples. The authors also wish to express their gratitude to the Filipino volunteers who gave generously of their time and provided the skin VOC samples.

\section{REFERENCES}

1. S. Riazanskaia, G. Blackburn, M. Harker, D. Taylor and C. L. P. Thomas, Analyst. 2008, 133, 1020.

2. S. K. Pandey and K. H. Kim, TRAC, 2011, 30, 784.

3. A. M. Curran, S. I. Rabin, P. A. Prada and K. G. Furton, J. Chem. Ecol. 2005, 31, 1607.

4. Y. Yokoyama, M. Aragalu, H. Sato and M. Tsuchiya, Analyt. Chim. Acta. 1991, 246, 405.

5. S. M. Shirreffs and R. J. Maughan, J. Appl. Physiol. 1997, 82, 336.

6. M. Gallagher, J. Wysocki, J. J. Leyden, A. I Spielmen, X. Sun and G. Preti, Br. J. Derm. 2008, 159, 780.

7. K. Nakagawa, D. Ibusuki, Y. Suzuki, S. Yamashita, O. Higuchi, S. Oikawa and T. Miyazawa, J. Lipid Res. 2007, 49, 2779.

8. H. A. Soini, K. E. Bruce, I. Klouckova, R. G. Brereton, D. J. Penn and M. V. Novotny, Anal. Chem. 2006, 78, 7161.

9. C. Bicchi, C. Cordero, E. Liberto, P. Rubiolo, B. Sgorbini, P. Sandra, J. Chromatgogr. A. 2007, 1148, 137.

10. S. Sisalli, A. Adao, M. Lebel, I. Le Fur, P. Sandra, LC-GC Eur. 2006, 19, 33.

11. U. R. Bernier, M. M. Booth, R. A. Yost, Anal. Chem. 1999, 71, 1. U. R. Bernier, M. M. Booth, R. A. Yost, Anal. Chem. 1999, 71, 1.

12. U.R. Bernier, D.L.Kline, D.R.Barnard, C.E.Schrek and R.A.Yost, Anal. Chem. 2000, 72, 747. 
13. D. J. Penn, E. Oberzaucher, K. Grammer, G. Fischer, H. A. Soini, D. Wiesler, M. V. Novotny, S. J. Dixon, Y. Xu and R. G. Brereton, J. R. Soc. Interface, 2007, 4, 331.

14. B. Sgorbini, M.R. Ruosi, C. Cordero, E. Liberto, P. Rubiolo and C. Bicchi, J. Chrom. A, 2010, 1217, 2599.

15. Z. M. Zhang, J. J. Cai, G. H. Ruan, G. K. Li, J. Chromatogr. B. 2005, 822, 244.

16. Y. Sekine, S. Toyooka and S. F. Watts, J. Chromatogr. B. 2007, 859, 201.

17. S. J. Dixon, Y. Xu, R. G. Brereton, H. A. Soini, M. V. Novotny, E. Oberzaucher, K. Grammer and D. J. Penn, Chemom. Intell. Lab. Syst. 2007, 87, 161.

18. A.N.Thomas, S. Riazanskaia, W. Cheung, Y. Xu, R. Goodacre, C.L.P.Thomas, M.S.Baguneid and A Bayat, Wound Rep. Reg. 2010, 18, 391.

19. T. Abaffy, R. Duncan, D. D. Riemer, O. Tietje, G. Elgart, C. Milikowski and R. A. DeFazio, Melanoma Volatile Biomarkers, 2010, 5, 11

20. K. Nose, T. Mizuno, N. Yamane, T. Kondo, H. Ohtani, S. Araki and T. Tsuda, Anal. Sci. 2005. 21, 1471.

21. A. M. Curran, P. A. Prada and K. G. Furton, J. Forensic Sci. 2010, 55, 50.

22. A. M. Curran, C. F. Ramirez, A. A. Schoon and K. G. Furton, J. Chrom. B, 2007, 846, 86.

23. X. Zeng, J. J. Leyden, A. I. Spielman and G. Preti, J. Chem. Ecol. 1996, 22, 237.

24. S. Haze, Y. Gozu, S. Nakamura, K. Sawano, H. Ohta and K. Yamazaki, J. Incest. Dermatol. 2001, 116, 520.

25. M. Troccaz, C. Starkenmann, Y. Niclass, M. Van de Waal and A. J. Clark, Chem. and Biodiv. 2004, 1, 1022.

26. X. Zeng, J. J. Leyden, H. J. Lawley, K. Sawano, I. Nohara and G. Preti, J. Chem. Ecol. 1991, 17, 1469.

27. A. Natsch, S. Derrer, F. Flachsmann and J. Schmid, Chem. and Biodiv. 2006, 3, 1.

28. Y. Hasegawa, M. Yabuki and M. Matsukane, Chem. and Biodiv. 2004, 1, 2042. 
29. M. McCulloch, T. Jezierski, M. Broffman, A. Hubbard, K. Turner and T. Janecki, Integr. Cancer Ther., 2006, 5, 30.

30. E. Moser, M. McCulloch, J. Vet. Behav, . 2010, 5, 145.

31. J. Cornu, G. Cancel-Tassin, V. Ondet, C. Girardet ,O. Cussenot, European Urology, 2011, 59, 197.

32. D. Pickel, G. P. Manucy, D. B. Walker, S. B. Hall, J. C. Walker, App. Animal Behav. Sci., 2004, 89, 107.

33. M. Katona, J. Denes, R. Skoumal, M. Toth and Z. Takats, Analyst, 2011, 136, 835.

34. P. Martinez-Lozano, Int. J. Mass Spectrom. 2009, 282, 128.

35. P. Martinez-Lozano and J. Fernandez de la Mora, J. Am. Soc. Mass Spectrom. 2009, 20, 1060

36. H. Chen, S. Yang, A. Wortmann and R. Zenobi, Angew. Chem. Int. Ed. 2007, 46, 7591.

37. C. Turner, B. Parekh, C. Walton, P. Španěl, D. Smith and M.Evans, Rapid Commun. Mass Spectrom., 2008, 22, 526.

38. M. M. L. Steeghs, B. W. M. Woeksops, K. van Swam, S. M. Cristescu, P. T. J. Scheepers and F. J. M. Harren, Int. J. Mass Spectrom. 2006, 253, 58.

39. J. C. Reynolds, G. J. Blackburn, C. Guallar-Hoyas, V. H. Moll, V. BocosBintintan, G. Kaur-Atwal, M. D. Howdle, E. L. Harry, L. J. Brown, C. S. Creaser and C. L. P. Thomas, Anal. Chem. 2010, 82, 2391.

40. M. Harker, A. M. Carvell, V. P. Marti, S. Riazanskaia, H, Kelso, D. Taylor, S. Grimshaw, D. S. Arnold, R. Zillmer, J. Shaw, J. M. Kirk, Z. M. Alcasid, S. Gonzales-Tanon, G. P. Chan, E. A. Rosing, and A. M. Smith, J Dermatol Sci. 2014, 73, 23.

41. A. Martin, M. Saathoff, F. Kuhn, H. Max, L, Terstegen and A. Natsch, J. Investigative Dermatology 2010, 130, 529-540.

42. G. Madalinski, E. Godat, S. Alves, D. Lesage, E. Genin, P. Levi, J. Labarre, J. Tabet, E. Ezan and C. Junot, Anal. Chem. 2008, 80, 3291

43. E. Hoffmann and V. Stroobant. "Mass Spectrometry: Principles and Applications" 3rd ed. 2007. Publ. Wiley-Interscience, ISBN-10: 0470033118.

44. T. Lang, C. Justenhoven, S. Winter, C. Baisch, U. Hamann, V. Harth, Y. Ko, S. Rabstein, A. Spickenheuer, B. Pesch, T. Brüning, M. Schwab and H. Brauch, Breast Cancer Res. Treat. 2011, 129,993-999. 


\section{TABLES}

Table 1. Total mass spectral count responses for the target VFAs from 10 participants with varying mean malodour scores (MMS).

\begin{tabular}{|c|c|c|c|c|c|}
\hline \multirow[b]{2}{*}{ Participant ID } & \multirow[b]{2}{*}{ MMS } & \multicolumn{4}{|c|}{ TD-ESI-MS Response (Counts) } \\
\hline & & C4-VFA & C5-VFA & C6-VFA & $3 \mathrm{M} 2 \mathrm{H}$ \\
\hline 1 & 4.75 & 234 & 483 & 636 & 413 \\
\hline 2 & 4.75 & 246 & 321 & 721 & 250 \\
\hline 3 & 4 & 216 & 354 & 824 & 264 \\
\hline 4 & 3.33 & 258 & 430 & 1247 & 0 \\
\hline 5 & 4 & 202 & 340 & 908 & 205 \\
\hline 6 & 1.25 & 93 & 129 & 339 & 0 \\
\hline 7 & 2.25 & 234 & 400 & 1223 & 0 \\
\hline 8 & 2.5 & 136 & 181 & 403 & 0 \\
\hline 9 & 1.33 & 97 & 207 & 242 & 0 \\
\hline 10 & 1.33 & 194 & 295 & 859 & 0 \\
\hline
\end{tabular}

Note. $\quad 2 M P$ : 2-methylpropanoic acid; B: butanoic acid; 3MB: 3-methylbuanoic acid; P: pentanoic acid; H: hexanoic acid; and 3M2H: 3-methyl, 2-hexanoic acid.

Table 2 TD-GC-MS chromatographic peak area responses for the target VFAs from the 10 participants detailed in Table 1.

\begin{tabular}{rrrrrrr}
\hline & \multicolumn{7}{c}{ Chromatographic Peak Area (AU) } \\
Participant ID & 2MP & B & 3MB & P & H & 3M2H \\
\hline 1 & 200 & 1287 & 878 & 863 & 364 & 688 \\
2 & 0 & 157 & 60 & 121 & 158 & 22 \\
3 & 52 & 1152 & 258 & 310 & 614 & 50 \\
4 & 17 & 68 & 50 & 26 & 470 & 0 \\
5 & 20 & 300 & 21 & 65 & 163 & 18 \\
6 & 0 & 106 & 27 & 109 & 545 & 0 \\
7 & 0 & 145 & 42 & 110 & 406 & 0 \\
8 & 30 & 88 & 59 & 92 & 332 & 0 \\
9 & 58 & 53 & 26 & 66 & 158 & 0 \\
10 & 0 & 41 & 27 & 59 & 213 & 0 \\
\hline
\end{tabular}

Note. $\quad 2$ MP: 2-methylpropanoic acid; B: butanoic acid; 3MB: 3-methylbuanoic acid; P: pentanoic acid; $\mathrm{H}$ : hexanoic acid; and 3M2H: 3-methyl, 2-hexanoic acid. 
Table 3 Additional VFAs from the skin of an MMS 4 individual identified by TD-ESI-MS and TD-GC-MS. Proposed identities are based on predicted elemental composition from accurate mass data (TD-ESI-MS), chromatographic retention time and NIST searching (TD-GC-MS).

\begin{tabular}{|c|c|c|c|c|c|c|c|}
\hline \multirow[b]{2}{*}{ ID } & \multirow[b]{2}{*}{$\mathrm{M}$} & \multicolumn{2}{|c|}{ TD-ESI-MS } & \multicolumn{4}{|c|}{ TD-GC-MS } \\
\hline & & {$[\mathrm{M}-\mathrm{H}]^{-}$} & $\begin{array}{c}\Delta \mathrm{M} \\
(\mathrm{ppm})\end{array}$ & $t_{r} / \min$ & $\begin{array}{l}\text { NIST } \\
\text { match }\end{array}$ & $\begin{array}{l}\text { Base } \\
\text { Peak }\end{array}$ & $\begin{array}{c}\Delta \mathrm{M} \\
(\mathrm{ppm})\end{array}$ \\
\hline Octanoic & 144.1150 & 143.1072 & 1.4 & 18.79 & 878 & 60.0211 & 6.7 \\
\hline Nonanoic & 158.1307 & 157.1229 & 6.4 & 21.59 & 807 & 60.0211 & 3.3 \\
\hline Decanoic & 172.1463 & 171.1385 & 7.0 & 24.62 & 901 & 60.0211 & 1.7 \\
\hline Undecanoic & 186.1620 & 185.1542 & 8.0 & 27.13 & 861 & 60.0211 & 8.3 \\
\hline Dodecanoic & 200.1776 & 199.1698 & 2.0 & 28.90 & 838 & 73.0290 & 2.7 \\
\hline Tridecanoic & 214.1933 & 213.1855 & 5.6 & 31.24 & 719 & 73.0290 & 8.2 \\
\hline Tetradecanoic & 228.2089 & 227.2011 & 1.8 & 33.94 & 849 & 73.0290 & 5.5 \\
\hline Pentadecanoic & 242.2246 & 241.2168 & 4.1 & 35.99 & 769 & 73.0290 & 4.1 \\
\hline
\end{tabular}




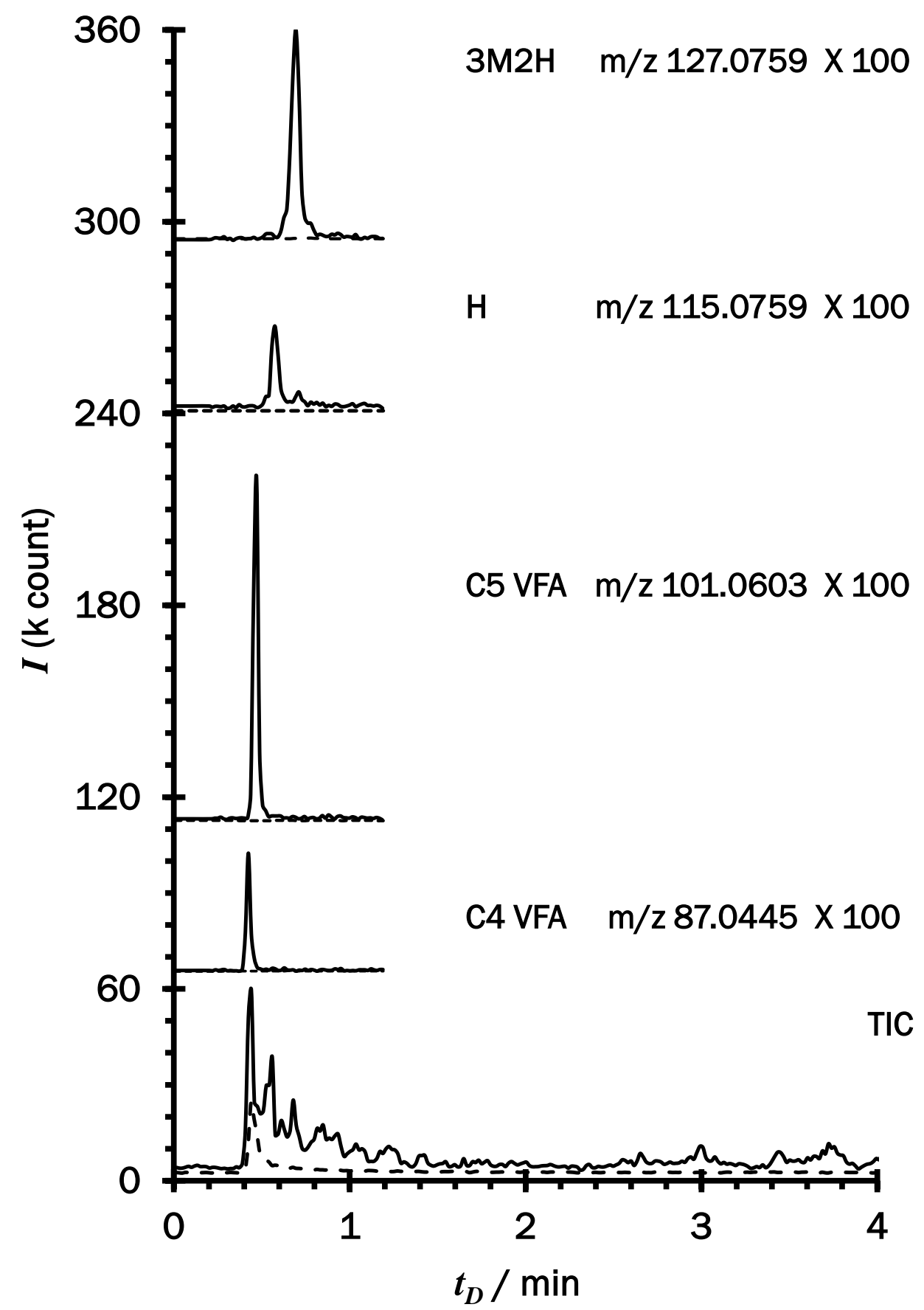

Figure 1. Key: 2MP: 2-methylpropanoic acid; B: butanoic acid; 3MB: 3-methylbuanoic acid; $\mathrm{P}$ : pentanoic acid; H: hexanoic acid; and 3M2H: 3-methyl, 2-hexanoic acid.

Bottom: An example of a total ion response profile obtained by TD-ESI-MS compared to the offset profiles obtained for C4 VFA, C5 VFA, $\mathrm{H}$ and $3 \mathrm{M} 2 \mathrm{H}$, at 100 times magnification. The dotted lines are the responses obtained from a blank skin patch. The sample was taken from an individual with a relatively high organoleptic score for VFA odour, MMS $=4$, that is a participant without the (SNP) $538 \mathrm{G} \rightarrow \mathrm{A}$. 


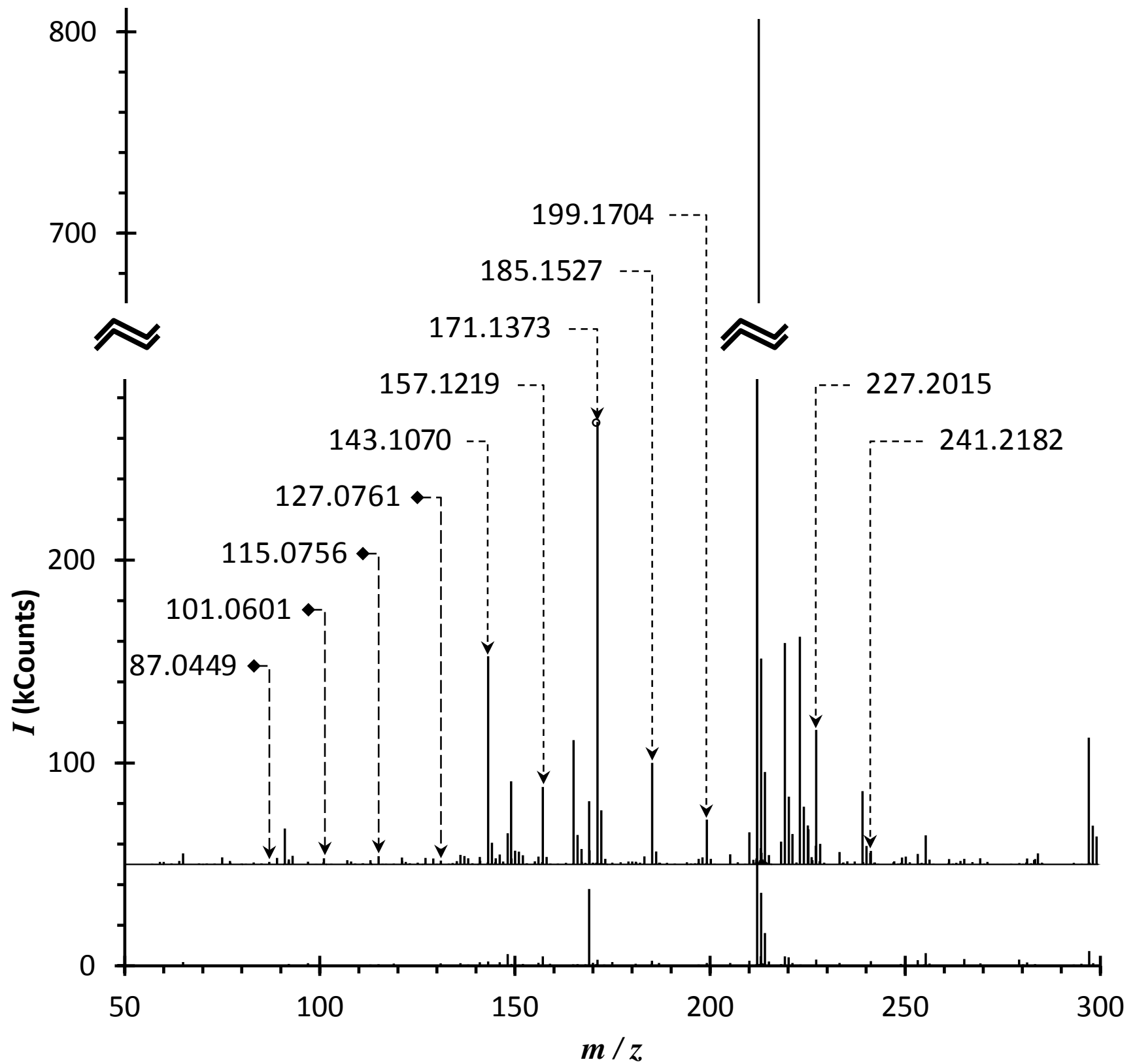

Figure 2. The negative mode mass spectra of the example thermal desorption profile shown in Figure 1. The n-butyl benzensulfonamide ion $(m / z$ 212.0745) was the most intense ion in the blank (bottom) and the sample (top.) As an internal lockmass this enabled identification of the VFA targets. From left to right the $m / z$ values used to assign these peaks were: 87.0449 , butanoic/2-methylpropanoic (C4 VFA 3.4ppm); 101.0601, pentanoic/ 3-methylbutanoic (2.0ppm); 115.0756, hexanoic (2.6ppm); and 127.0761, 3-methyl-2-hexenoic acid (1.6ppm). Other signals attributable to higher molecular weight VFAs were also observed see Table 3. 


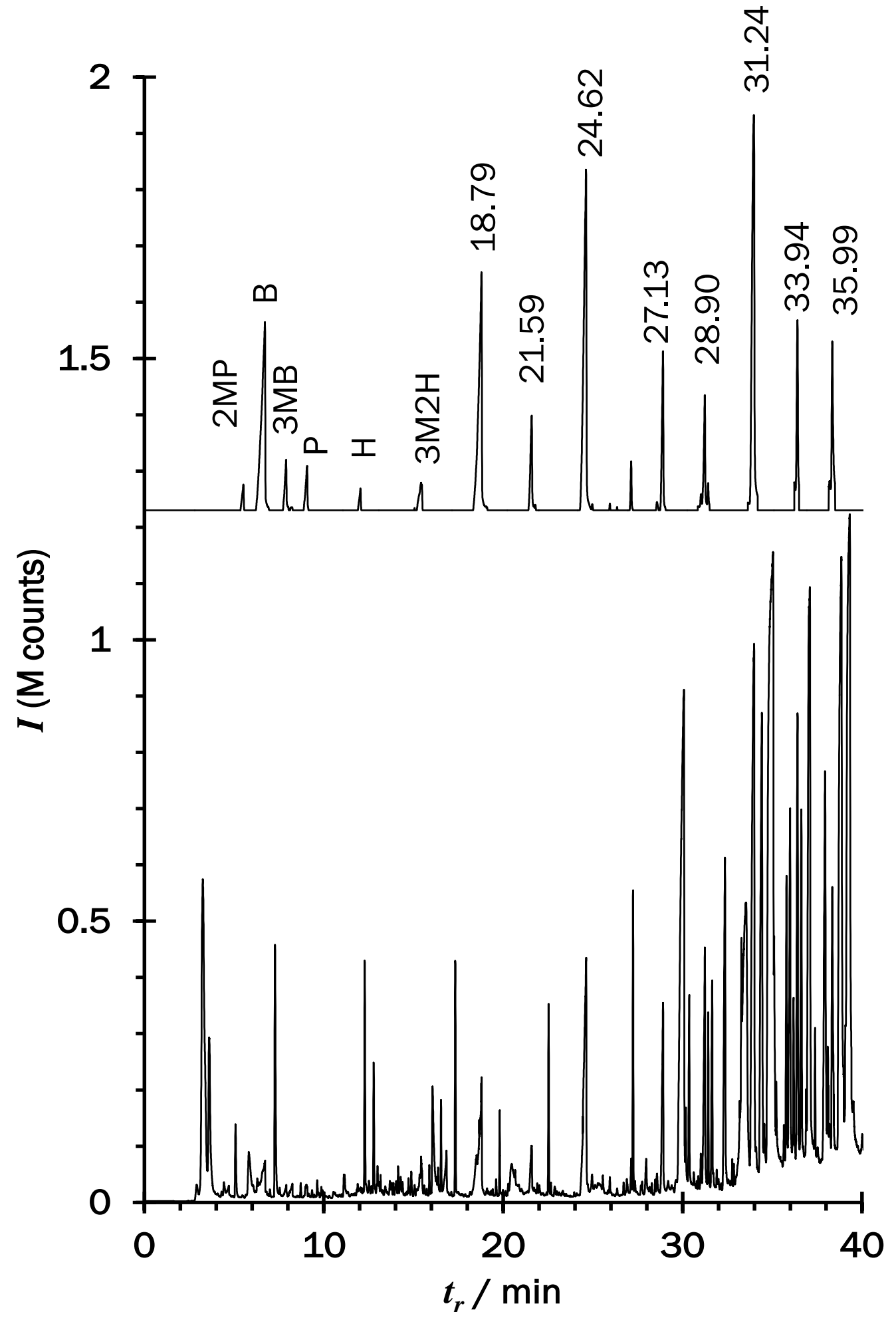

Figure 3. Bottom: The first 40 min TD-GC-MS TIC of a skin sample from the same individual presented in Figures 1 and 2. Top: an offset composite selected ion chromatogram at X10 magnification where: 2MP, 2-methylpropanoic acid; $B$, butanoic acid; 3MB, 3-methylbutanoic acid; $\mathrm{P}$, pentanoic acid; $\mathrm{H}$, hexanoic acid; and $3 \mathrm{M} 2 \mathrm{H}$ 3-methyl-2-hexenoic acid. Other peaks from $t_{r}=18.79$ to $35.99 \mathrm{~min}$ were attributed to higher molecular weight VFAs see Table 3. 

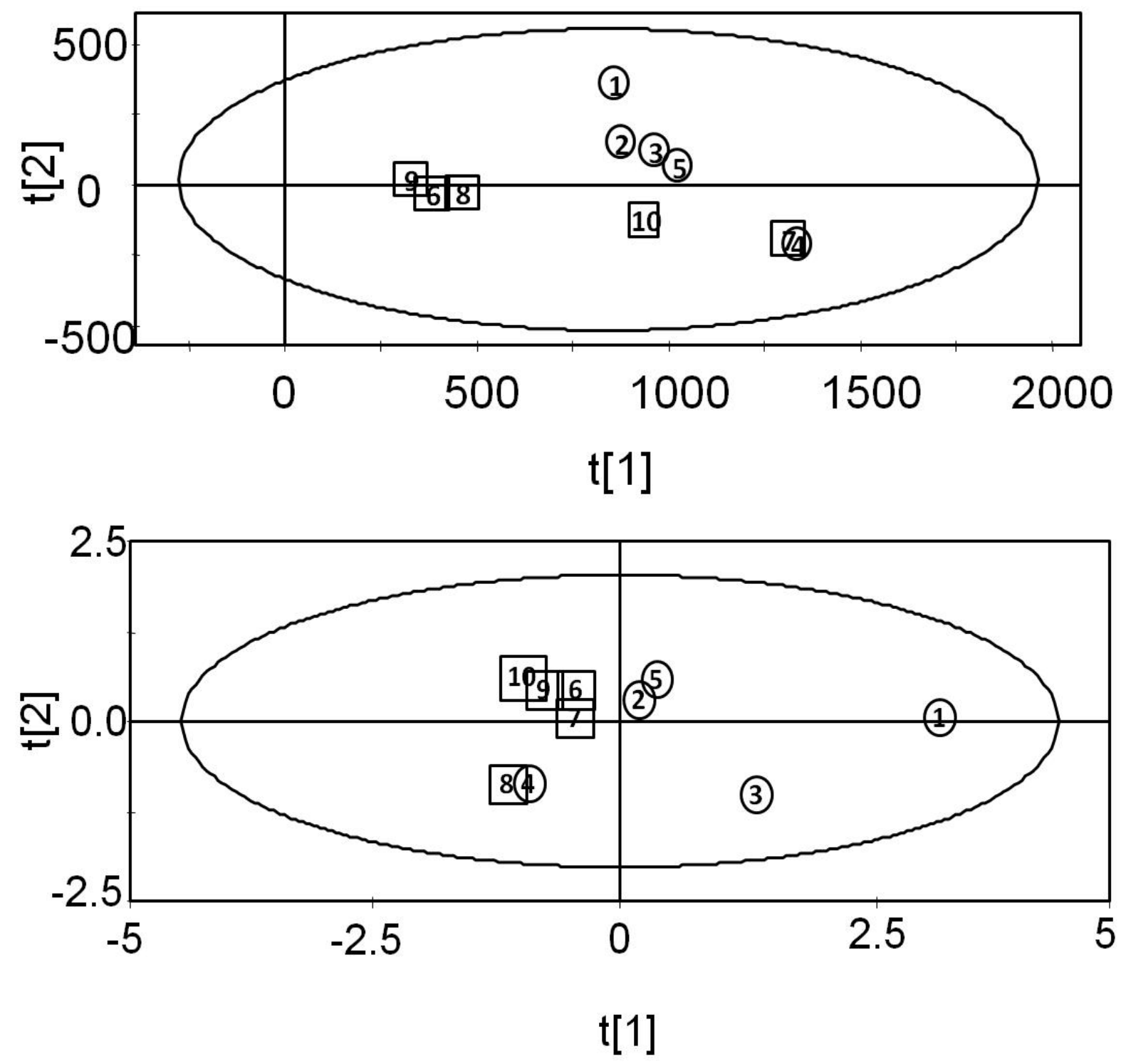

Figure 4. Unsupervised principle component analysis (PCA) of the target compounds. Top 5 high odour (circles) and 5 low odour (squares) observations analysed by thermal desorption-negative mode electrospray ionisation mass spectrometry and $[B]$ the same set of targets and observations analysed by gas chromatography mass spectrometry. Both data sets show separation between the two classes with observation 4 being misclassified as low odour by both analytical techniques. 

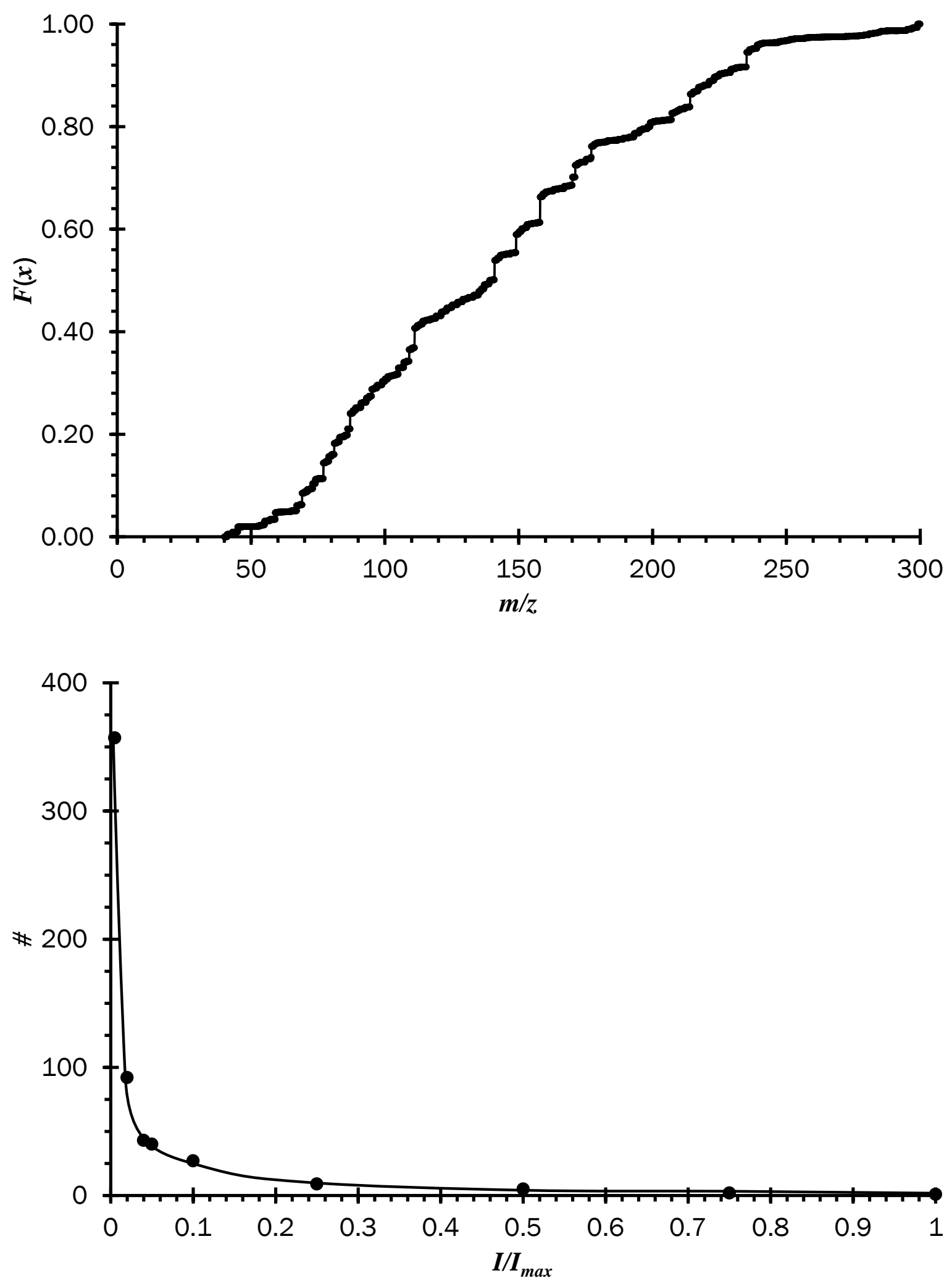

Figure 5. Top. An example cumulative distribution function of negative mode TD-SESI-MS responses showing the distribution of chemical information across the range $m / z$ 40 to $\mathrm{m} / \mathrm{z}$ 300. The An example of a normalised distribution of intensities for the 598 features observed in the negative mode showing the abundance of low intensity features; comparable with exhaled breath VOC profiles. 